\section{Der repetitive Waschtest als Modell zur Beurteilung von Hautschutz- präparaten am Beispiel einer dexpanthenolhaltigen Formulierung}

Zusammenfassung. Der repetitive Waschtest mit NLS stellt ein Irritationsmodell dar, mit dem Hautschutzpräparate auf ihre Wirksamkeit gegenüber hydrophilen Reizstoffen überprüft werden können. An eine repetitive Waschphase mit vorheriger Anwendung der Prüfpräparate schließt sich eine Behandlungsphase mit alleiniger Applikation der Emulsionen ohne weitere Waschungen an. In diesem Modell wurde ein hydrophiles Vehikel mit und ohne Dexpanthenol getestet. Dabei konnte ein hydratisierender und antiinflammatorischer Effekt von Dexpanthenol nachgewiesen werden.

Evaluation of a Dexpanthenolcontaining Formulation in the Repetitive Washing Test as a Model for the Investigation of Barrier Creams. The repetitive washing test with SLS represents an irritation model for the evaluation of the efficacy of barrier creams against hydrophilic irritants. Subsequently to the washing period with previous application of the barrier cream a treatment period without any washings followed. In this model a hydrophilic emulsion with and without Dexpanthenol was investigated. The results demonstrated a hydrating and an anti-inflammatory effect of Dexpanthenol.

Jeder Kontakt zu waschaktiven Substanzen führt an der Haut zu einer Störung der epidermalen Barrierefunktion $[8,19]$, die sich als Anstieg des transepidermalen Wasserverlustes und als Reduktion der Hornschichtfeuchtigkeit dokumentieren lässt [11]. Darüber hinaus kommt es zur Entfettung der Hautoberfläche $[9,10]$. Repetitive Waschungen bringen einen kumulativ toxischen Effekt mit sich, der individuell unterschiedlich in eine irritativ-toxische Kontaktdermatitis einmündet [15], die die Entwicklung einer späteren allergischen Kontaktdermatitis begünstigen kann [12]. Damit hat der Kontakt zu Waschaktivsubstanzen eine große gesundheitsökonomische Bedeutung mit ganz besonderer Relevanz in der Berufsdermatologie.

Von Hautschutzpräparaten wird eine Vermeidung oder Reduktion des irritativen Wascheffektes erwartet. Ein zusätzlicher

Akt Dermatol 2001; 27: 279-284

(c) Georg Thieme Verlag Stuttgart · New York ISSN 0340-2541
W. Gehring, M. Gloor

Hautklinik am Klinikum der Stadt Karlsruhe gGmbH

(Direktor: Prof. Dr. M. Gloor)
Vorteil wäre es, wenn das Hautschutzpräparat aufgrund seiner Zusammensetzung die Repairmechnismen der irritativ geschädigten epidermalen Barriere unterstützen könnte. Vor diesem Hintergrund haben wir am Beispiel eines hydrophilen Vehikels mit Dexpanthenol als Inhaltsstoff im Rahmen einer doppelblinden, vehikelkontrollierten, randomisierten Studie den repetitiven Waschtest auf seine Aussagekraft hin untersucht.

\section{Material und Methodik}

\section{Prinzip des repetitiven Waschtestes}

Das Prinzip des repetitiven Waschtestes ist es, den toxisch irritativen Einfluss einer Waschung $\mathrm{zu}$ standardisieren und $\mathrm{zu}$ maximieren. Damit werden vergleichende Untersuchungen bei einem Status möglich, der der Situation nahe kommt, die sich beim häufigen Kontakt der Haut zu waschaktiven Substanzen einstellt. Dieses Testverfahren eignet sich ganz besonders für die Prüfung und Beurteilung von Hautschutzpräparaten (barrier creams), von denen eine protektive Wirkung gegenüber hydrophilen Reizstoffen erwartet wird.

\section{Durchführung des repetitiven Waschtestes}

Die Untersuchungen erfolgen an der Volarseite beider Unterarme, die zuvor mit einem nicht abwaschbaren Marker in zwei Quadranten eingeteilt werden. Somit entstehen an beiden Armen insgesamt 4 Testfelder, die jeweils eine Größe von etwa $24 \mathrm{~cm}^{2}(6 \mathrm{~cm} \times 4 \mathrm{~cm})$ aufweisen (Abb.1).

Die Waschungen werden an 7 aufeinander folgenden Tagen 5mal täglich mit 0,01n NLS durchgeführt. Durch den Versuchsablauf bedingt wird am 1 . und letzten Tag des Waschtestes nur 3-mal gewaschen. Zur Standardisierung des Waschvorganges benutzen die Probanden einen Schaumstoffroller, der eine Schwammwalze mit einem Durchmesser von 3,5 cm und einer Länge von $5 \mathrm{~cm}$ trägt. Der Hohlstiel des Rollers ist mit Blei ausgefüllt, so dass der Schaumstoffroller ein Gewicht von $200 \mathrm{~g}$ besitzt. Für die Waschung taucht man den Schwamm in die Waschlösung. Anschließend wird die Rolle mit dem Auflagedruck ihres Eigengewichtes 50-mal über die Testfelder hin und her gerollt. Im Anschluss daran werden die Unterarme in lauwarmem Wasser kurz abgewaschen, um Tensidreste von der Hautoberfläche zu entfernen. Danach werden die Unterarme mit einem fusselfreien Tuch vorsichtig getrocknet. Inter- 


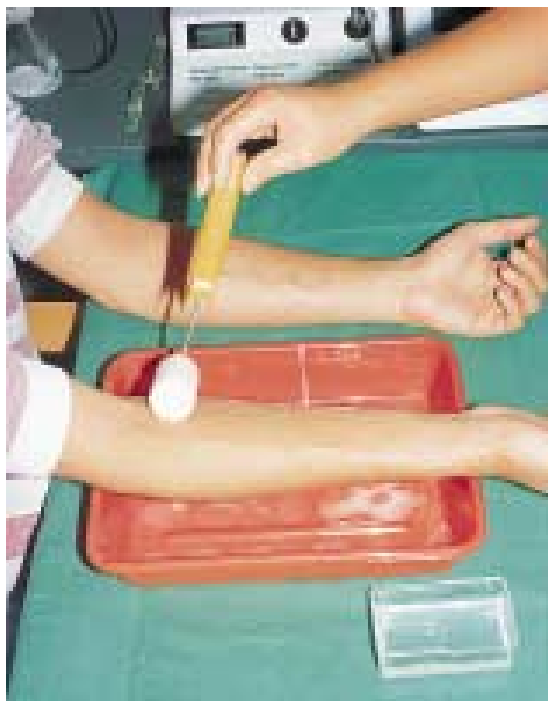

Abb. 1 Durchführung des standardisierten Waschvorganges mit einem Schaumstoffroller.

mittierend zu den Waschungen können Prüfpräparate appliziert werden, deren Anwendung über die siebentägige Waschphase hinaus fortgesetzt werden kann.

\section{Anwendung der Prüfpräparate intermittierend zu den Waschungen}

Etwa 30 min vor den Waschungen kamen jeweils $50 \mu l$ der Prüfpräparate auf den entsprechenden Testfeldern zur Anwendung. Zur Erleichterung der Dosierung waren die Testsubstanzen in durchsichtigen $1 \mathrm{ml}$-Plastikspritzen mit Skalierung abgefüllt. Ein Teilstrich entspricht $10 \mu \mathrm{l}$. Diese Menge sollte gleichmäßig durch leichte Massagebewegungen auf das gesamte Testfeld verteilt werden. Dabei waren die Probanden gehalten, bei jedem Prüfpräparat einen anderen Finger zu verwenden, um Vermischungen der Testformulierungen zu vermeiden. Ein Testfeld wurde zur Kontrolle nur gewaschen und blieb ansonsten unbehandelt.

\section{Behandlungsphase nach Beendigung der repetitiven Waschungen}

An die Waschphase schloss sich ein 4-tägiger Behandlungszyklus ohne weitere Irritation durch Waschung an. Während dieser Periode kamen die Prüfpräparate $3 \times$ tgl. zur Anwendung. In dieser Nachbehandlungsphase können Repair-Mechanismen erfasst werden.

\section{Überblick über den Versuchsablauf}

Aus praktischen Gründen begannen die Untersuchungen am Montag, so dass der gesamte Versuch zeitlich folgendermaßen ablief (Tab.1):

\section{Beurteilung der Testreaktionen}

Vor der ersten Anwendung der Prüfpräparate und der ersten Waschung wurden in jedem Feld die Hornschichtfeuchtigkeit mit dem Corneometer CM 820 (Courage und Khasaka) und der transepidermale Wasserverlust mit dem Tewameter TM 210 (Courage und Khasaka) als Ausgangswert bestimmt. Die Verlaufsmessungen wurden am 5., 8., 9. und 12. Tag vorgenommen.

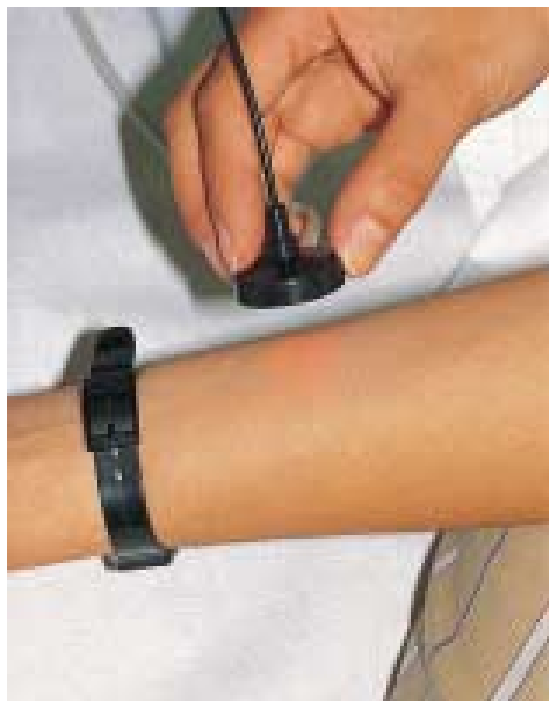

Abb. 2 Beurteilung einer Testreaktion mit der Laser-Doppler-Flowmetrie. Der Messkopf wird in direkten Kontakt zur Haut gebracht.

Das Messprinzip der Corneometrie basiert auf einer kapazitiven Bestimmung der unterschiedlichen Dielektrizitätskonstanten von Wasser und anderen Stoffen [20].

Der transepidermale Wasserverlust gilt als ein wichtiger Parameter für die epidermale Barrierefunktion. Das Messprinzip beruht darauf, dass ein hautnah aufgebrachter Messkopf zwei Fühlerpaare enthält, von denen eines die Feuchtigkeit und das andere die Temperatur misst. Daraus ermittelt ein im Gerät integrierter Kleincomputer den Partialdruck des Wasserdampfes für die zwei verschiedenen übereinander liegenden Ebenen jedes Fühlerpaares und über den Gradienten des Partialdruckes die Verdunstungsrate. Die Messwerte drücken die Verdunstungsrate in $\mathrm{g} / \mathrm{m}^{2} \mathrm{~h}$ aus [17].

Als Parameter der durch den Waschvorgang hervorgerufenen Entzündungsreaktion galt die Laser-Doppler-Flowmetrie (Periflux, Fa. Servomed) (Abb. 2).

Die LDF spiegelt die Hautdurchblutung wider und ist zur Beurteilung aller Faktoren geeignet, von denen ein Einfluss auf die Hautdurchblutung zu erwarten ist, und ist somit geeignet, Entzündungsreaktionen zu beurteilen. Dabei tritt ein Laserstrahl niedriger Leistung in die Haut ein. Es handelt sich um einen Helium-Neon-Laser der Wellenlänge 632,8 nm mit einer Ausgangsleistung von $2 \mathrm{~mW}$. Der Brenn- und Messpunkt des Laserstrahles liegt in 0,5-1,0 mm Tiefe. Die Messung basiert auf dem Prinzip der Doppler-Verschiebung, d.h. der Frequenzbzw. Wellenlängenänderung, wenn das Licht von vorbeiströmenden Teilchen reflektiert wird. In diesem Fall spiegelt die LDF die Strömungsgeschwindigkeit der Erythrozyten wider und gibt aufgrund ihrer Eindringtiefe Aufschluss über den relativen Grad der Durchblutung bis etwa zum mittleren Corium [16].

Für weitere methodische Einzelheiten verweisen wir auf die Literatur $[1,3,18]$. 
Tab. 1 Zeitplan des Versuchsablaufs

\begin{tabular}{llllll}
\hline & Montag & Freitag & Montag & Dienstag & Freitag \\
\hline $\begin{array}{l}\text { vormittags } \\
\text { nachmittags }\end{array}$ & Messung* & Messung** & Messung** & Messung* $^{*}$ & Messung* $^{*}$ \\
\hline
\end{tabular}

* Behandlung ohne Waschung; ${ }^{* *}$ Behandlung mit Waschung

\section{Prüfpräparate}

Zur Verfügung standen 3 Prüfformulierungen ${ }^{1}$ :

1. Vehikel

2. Vehikel plus 2,5\% Dexpanthenol

(entspricht Bepanthol ${ }^{\circledR}$ Roche Handbalsam)

3. Vehikel plus 5,0\% Dexpanthenol

4. ein Testfeld wurde nur gewaschen und diente unbehandelt als Kontrolle

Die Zusammensetzung des Vehikels ist nach INCI folgendermaßen deklariert:

Aqua, Octyldodecanol, Propylene Glycol Dicaprylate/Dicaprate, Cetyl Alcohol, Glyceryl Stearate, Stearyl Alcohol, Ceteareth12, Ceteareth-20, Dimethicone, Pantolactone, Parfum, Chlorhexidine Dihydrochloride.

\section{Probandenkollektive}

In die Studie wurden 20 gesunde Probanden mit einem Mindestalter von 18 Jahren nach dem Zufallsprinzip eingeschlossen. Akute Hauterkrankungen oder die Verwendung von Externa im Bereich der Testareale innerhalb der letzten 3 Wochen galten als Ausschlusskriterium. Ebenso konnten schwangere und stillende Frauen nicht an der Studie teilnehmen. Alle Probanden waren über den Testablauf genau unterrichtet und hatten ihr ausdrückliches Einverständnis erklärt. Vor Beginn der Studie ist das Votum der Ethik-Kommission der Landesärztekammer Baden-Württemberg eingeholt worden.

Die Prüfpräparate kamen bei folgendem Kollektiv zur Anwendung (Tab. 2):

Tab. 2 Alter- und Geschlechtsverteilung der Probanden

\begin{tabular}{llll} 
& $N$ & Durchschnittsalter & Altersgrenzen \\
\hline Männer & 3 & 29,7 Jahre & 18-46 Jahre \\
Frauen & 16 & 34,6 Jahre & 24-58 Jahre \\
\hline
\end{tabular}

Ein Proband hat ohne Nennung von Gründen die Studie vorzeitig abgebrochen.

\footnotetext{
${ }^{1}$ Die Prüfpräparate wurden von Roche Nicholas, Deutschland GmbH zur Verfügung gestellt.
}

\section{Statistische Auswertung}

Die Ergebnisse wurden nach vorheriger Varianzanalyse mit dem Friedman-Test nach dem Wilcoxon-Test für verbundene Stichproben statistisch ausgewertet. Die Darstellung der Ergebnisse in den Abbildungen bezieht sich auf die Mittelwerte. Zur genauen Charakterisierung unserer Daten werden Mittelwerte, Standardabweichung, Median und BOX tabellarisch angegeben (Tab. 3-5).

\section{Ergebnisse}

\section{Hornschichtfeuchtigkeit}

An unbehandelter Haut hat der repetitive Waschvorgang zu einem starken Verlust an Hornschichtfeuchtigkeit geführt, der auch am Ende der Nachbehandlungsphase ohne weitere Waschanwendungen noch nicht kompensiert war. Alle Prüfformulierungen haben der Austrocknung durch NLS entgegengewirkt. Sowohl während der Waschphase als auch im Nachbehandlungsintervall heben sich 2,5\% und 5\% Dexpanthenol statistisch signifikant vom Vehikel ab. Damit ist ein Wirkungsnachweis für Dexpanthenol erbracht und die aus den vorherigen Untersuchungen bekannte hydratisierende Wirkung von Dexpanthenol bestätigt (Abb. 3, Tab.3).

Statistische Unterschiede zwischen den beiden Konzentrationen sind nicht erkennbar. Jedoch heben sich tendenziell und gemessen am Signifikanzniveau gegenüber dem Vehikel 5\% gegenüber $2,5 \%$ vorteilhaft ab.

\section{Transepidermaler Wasserverlust}

An unbehandelter Haut hat der repetitive Waschvorgang zu einem starken Anstieg des TEWL geführt, der die gestörte epidermale Barrierefunktion widerspiegelt. Sofort nach Ende der Waschungen beginnt auch an unbehandelter, nur gewaschener Haut eine Restitution. Jedoch ist am Ende der Nachbehandlungszeit das Ausgangsniveau noch nicht wieder erreicht. Während des gesamten Testes - sowohl während der repetitiven Waschungen als auch beim Nachbehandlungsintervall - wurde der TEWL durch alle Prüfpräparate reduziert. Ähnlich wie bei der Beeinflussung der Hornschichtfeuchtigkeit wurde - auch gemessen am TEWL - ein Wirkungsnachweis für Dexpanthenol erbracht. Dabei unterscheiden sich beide Konzentrationen nicht voneinander; jedoch heben sich auch hier 5\% Dexpanthenol gegenüber 2,5\% tendenziell und gemessen am Signifikanzniveau gegenüber dem Vehikel vorteilhaft ab. Das Ergebnis spiegelt einen stabilisierenden Effekt von Dexpanthenol auf die epidermale Barrierefunktion wider (Abb. 4, Tab. 4). 
Tab. 3 Effekt von Dexpanthenol im repetitiven Waschtest - Corneometer (Differenzwerte zum Status vor Behandlung [T0])

\begin{tabular}{|c|c|c|c|c|c|c|}
\hline Corneometer & Tag & MW & ST & Median & Box & $\mathrm{N}$ \\
\hline \multirow[t]{4}{*}{ Vehikel } & T5 & $-1,23$ & 8,52 & $-2,2$ & $-6,6 / 4,4$ & 19 \\
\hline & $\mathrm{T} 8$ & $-2,18$ & 6,06 & $-2,2$ & $-5 / 2,2$ & 19 \\
\hline & T9 & $-1,7$ & 6,96 & $-2,1$ & $-9,2 / 2$ & 18 \\
\hline & $\mathrm{T} 12$ & $-4,04$ & 8,33 & $-4,6$ & $-10,6 / 1$ & 19 \\
\hline \multirow[t]{4}{*}{ Dexpanthenol 2,5\% } & T5 & $-0,8$ & 8,4 & $-1,8$ & $-5,4 / 5$ & 19 \\
\hline & $\mathrm{T} 8$ & 1,46 & 7,64 & 0 & $-4,8 / 7,4$ & 19 \\
\hline & T9 & 1,93 & 8,05 & 3,2 & $-5 / 8,8$ & 18 \\
\hline & $\mathrm{T} 12$ & 0,88 & 8,17 & $-1,2$ & $-5,2 / 8,8$ & 19 \\
\hline \multirow[t]{4}{*}{ Dexpanthenol 5\% } & T5 & 1,66 & 9,7 & 0,6 & $-3 / 10,2$ & 19 \\
\hline & T8 & 2,05 & 7,96 & 1 & $-2 / 6,2$ & 19 \\
\hline & T9 & 3,63 & 8,08 & 3,9 & $-0,6 / 11,6$ & 18 \\
\hline & $\mathrm{T} 12$ & 1,9 & 9,48 & 0,6 & $-2,6 / 10,6$ & 19 \\
\hline \multirow[t]{4}{*}{ unbehandelt } & T5 & $-7,2$ & 6,64 & $-6,6$ & $-12,2 /-5$ & 19 \\
\hline & $\mathrm{T} 8$ & $-10,3$ & 8,42 & -10 & $-14 /-6,6$ & 19 \\
\hline & T9 & $-10,91$ & 11,26 & $-10,3$ & $-19 /-4$ & 18 \\
\hline & $\mathrm{T} 12$ & $-11,72$ & 13,53 & $-8,6$ & $-20,8 /-5$ & 19 \\
\hline
\end{tabular}

Tab. 4 Effekt von Dexpanthenol im repetitiven Waschtest - Tewameter (Differenzwert zum Status vor Behandlung [T0])

\begin{tabular}{|c|c|c|c|c|c|c|}
\hline Tewameter & Tag & MW & ST & Median & Box & $\mathrm{N}$ \\
\hline \multirow[t]{4}{*}{ Vehikel } & T5 & 3,27 & 2,25 & 3,3 & $1,9 / 4,6$ & 19 \\
\hline & T8 & 4,36 & 3,22 & 5,3 & $2,2 / 7,2$ & 19 \\
\hline & T9 & 4,03 & 2,71 & 4,5 & $2,6 / 6,3$ & 18 \\
\hline & $\mathrm{T} 12$ & 2,73 & 1,99 & 2,8 & $1,5 / 4,3$ & 19 \\
\hline \multirow[t]{4}{*}{ Dexpanthenol 2,5\% } & T5 & 2,93 & 2,36 & 2,2 & $0,9 / 4,8$ & 19 \\
\hline & T8 & 3,99 & 3,33 & 3 & $2,1 / 6$ & 19 \\
\hline & T9 & 3,01 & 2,51 & 2,5 & $1,1 / 4,1$ & 18 \\
\hline & $\mathrm{T} 12$ & 2,1 & 1,8 & 1,7 & $1,3 / 3,2$ & 19 \\
\hline \multirow[t]{4}{*}{ Dexpanthenol 5\% } & T5 & 2,93 & 3,49 & 2,2 & $1,1 / 3,5$ & 19 \\
\hline & $\mathrm{T} 8$ & 3,21 & 3,45 & 3,2 & $1,4 / 3,8$ & 19 \\
\hline & T9 & 2,51 & 2,8 & 2,55 & $1 / 3,3$ & 18 \\
\hline & $\mathrm{T} 12$ & 1,94 & 1,89 & 1,4 & $0,9 / 3,1$ & 19 \\
\hline \multirow[t]{4}{*}{ unbehandelt } & T5 & 6,27 & 6,57 & 3,3 & $1,9 / 9,6$ & 19 \\
\hline & $\mathrm{T} 8$ & 9,55 & 7,96 & 6,8 & $2,9 / 16,4$ & 19 \\
\hline & T9 & 7,32 & 4,71 & 7,45 & $4 / 11,2$ & 18 \\
\hline & $\mathrm{T} 12$ & 4,52 & 2,37 & 4,2 & $2,2 / 6,8$ & 19 \\
\hline
\end{tabular}

\section{Laser-Doppler-Flowmetrie}

An unbehandelter Haut hat der repetitive Waschvorgang zu einer starken Erhöhung der Hautdurchblutung geführt, die die Entzündungsreaktion widerspiegelt. Sofort nach Ende der Waschungen beginnt auch an unbehandelter, nur gewaschener Haut eine Restitution. Jedoch ist ähnlich wie beim Verlauf des TEWL am Ende der Nachbehandlungszeit das Ausgangsniveau noch nicht wieder erreicht. Während des gesamten Testes sowohl während der repetitiven Waschungen als auch beim Nachbehandlungsintervall - wurde die Entzündungsreaktion durch alle Prüfpräparate reduziert. Dabei wurde wiederum ein Wirkungsnachweis für Dexpanthenol erbracht. Dexpanthenol hat aufgrund der vorliegenden Beobachtung einen anti- entzündlichen Effekt. Diesbezüglich scheinen $5 \%$ gegenüber $2,5 \%$ eindeutig überlegen zu sein. Nur am 8 . Tag, am Ende des Waschtestes, waren beide Konzentrationen gegenüber dem Vehikel statistisch signifikant. In der Nachbehandlungsphase waren nur noch 5\% Dexpanthenol vom Vehikel statistisch signifikant abgrenzbar. Am 9. Tag unterschieden sich sogar 5\% von 2,5\% signifikant voneinander (Abb. 5, Tab. 5).

\section{Diskussion}

Der repetitive Waschtest stellt ein Irritationsmodell dar, mit dem das individuelle Ekzemrisiko einschätzbar ist [8], mit dem aber auch Hautschutzpräparate, die vor den Waschungen aufgetragen werden, auf ihre Wirksamkeit hin gegenüber hy- 
Tab. 5 Effekt von Dexpanthenol im repetitiven Waschtest - Laser-Doppler-Differenzwerte zum Status vor Behandlung (T0)

\begin{tabular}{|c|c|c|c|c|c|c|}
\hline Laser-Doppler & Tag & MW & ST & Median & Box & $\mathrm{N}$ \\
\hline \multirow[t]{4}{*}{ Vehikel } & T5 & 7,6 & 9,4 & 4 & $2 / 13$ & 19 \\
\hline & $\mathrm{T} 8$ & 19 & 38,9 & 6 & $2 / 20$ & 19 \\
\hline & T9 & 10,7 & 17,4 & 2,5 & $0 / 19$ & 18 \\
\hline & $\mathrm{T} 12$ & 6,7 & 9,8 & 3 & $0 / 10$ & 19 \\
\hline \multirow[t]{4}{*}{ Dexpanthenol 2,5\% } & T5 & 6,1 & 7,5 & 4 & $0 / 10$ & 19 \\
\hline & $\mathrm{T} 8$ & 9,2 & 16,3 & 5 & $0 / 11$ & 19 \\
\hline & T9 & 6,2 & 6,9 & 4,5 & $3 / 9$ & 18 \\
\hline & $\mathrm{T} 12$ & 4,6 & 6,8 & 2 & $0 / 9$ & 19 \\
\hline \multirow[t]{4}{*}{ Dexpanthenol 5\% } & T5 & 6,8 & 10,8 & 4 & $0 / 11$ & 19 \\
\hline & $\mathrm{T} 8$ & 6,4 & 10,7 & 4 & $1 / 10$ & 19 \\
\hline & T9 & 4,1 & 7,3 & 3 & $-2 / 10$ & 18 \\
\hline & $\mathrm{T} 12$ & 2,7 & 7,7 & 0 & $-3 / 6$ & 19 \\
\hline \multirow[t]{4}{*}{ unbehandelt } & T5 & 21,6 & 33,5 & 6 & $2 / 27$ & 19 \\
\hline & $\mathrm{T} 8$ & 45,9 & 61,1 & 14 & $2 / 68$ & 19 \\
\hline & T9 & 16,4 & 22,2 & 8 & $4 / 27$ & 18 \\
\hline & T12 & 9,9 & 8,7 & 9 & $6 / 16$ & 19 \\
\hline
\end{tabular}

drophilen Reizstoffen überprüft werden können. Der Waschtest zielt darauf ab, eine Situation zu simulieren, wie sie beim täglichen Umgang mit Waschaktivsubstanzen im häuslichen Bereich und am Arbeitsplatz gegeben ist. Damit unterscheidet sich dieses Verfahren grundlegend von Testmodellen, bei denen die Waschaktivsubstanz und zu überprüfende Hautschutzpräparate okklusiv aufgetragen werden [4,5].

Durch die intermittierenden Anwendungen der Prüfpräparate zwischen den Waschungen des repetitiven Waschtestes können bereits Repairmechanismen durch die Prüfpräparate in den Test mit eingehen. Die Nachbehandlungsphase ohne weitere Irritation durch Waschung ist ausschließlich darauf ausgerichtet, Repairmechanismen zu erfassen. Der Waschtest hat sich anhand der untersuchten Präparate als geeignet erwiesen, eine Hautschutzwirkung zu untersuchen und zu dokumentieren. Bereits das Vehikel allein war effektiv. Auf diese Tatsache haben wir bei älteren Untersuchungen schon hingewiesen [2]. Insbesondere bestätigt sich unsere Beobachtung, dass auch ein hydrophiles Vehikel Schutz vor einem hydrophilen Reizstoff bieten kann. Die damalige Beobachtung bezieht sich auf hydrophile Emulsionen mit Urea oder Glycerin oder einer Kombination von beiden [13]. Bei der vorliegenden Studie konnte eine diesbezügliche Schutzwirkung durch ein hydrophiles Vehikel ohne Harnstoff oder Glycerin erreicht werden.

Der günstige Effekt des Vehikels konnte durch Dexpanthenol noch gesteigert werden. Damit ist ein Wirkungseffekt für Dexpanthenol erbracht, der vorausgegangene Untersuchungen bestätigt, bei denen wir für Dexpanthenol einen hydratisierenden Einfluss und eine stabilisierende Wirkung auf die epidermale Barrierefunktion festgestellt haben [7].

Besonders bemerkenswert sind die Ergebnisse der LaserDoppler-Flowmetrie, da sie eine antientzündliche Wirkung von Dexpanthenol belegen. Ein derartiger Effekt von Dexpanthenol hatte sich bereits bei einer vorausgegangenen Untersuchung angedeutet, die allerdings methodisch nicht ganz mit der vorliegenden Studie vergleichbar ist [5]. Die vorausgegan-

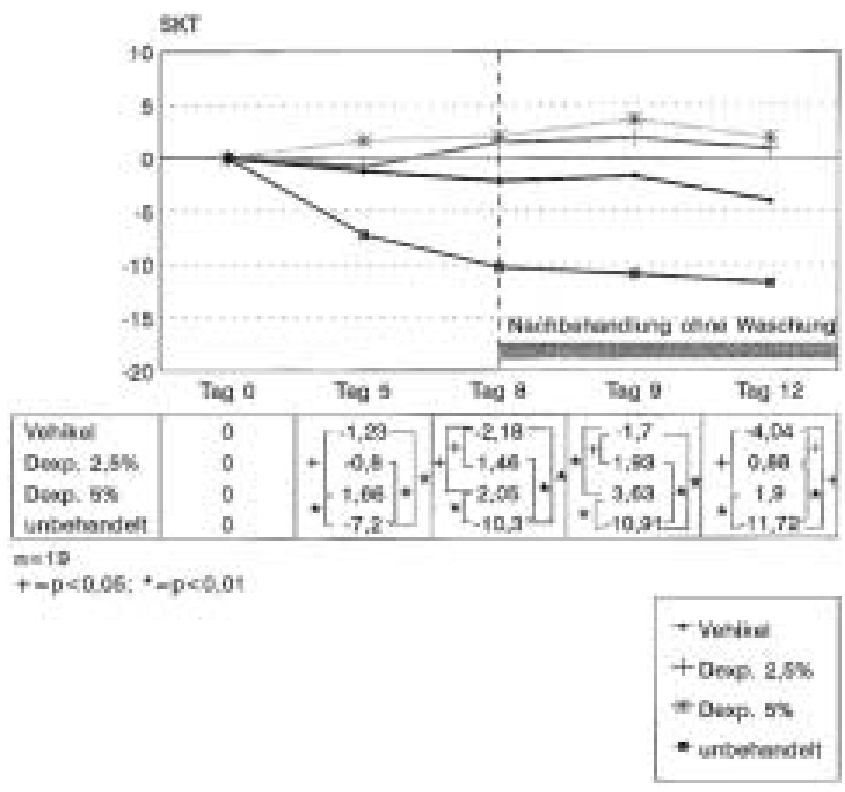

Abb.3 Hydratisierender Effekt von Dexpanthenol (Corneometer).

gene Untersuchung unterscheidet sich dadurch, dass die Prüfpräparate nicht prophylaktisch, sondern nach den Waschungen aufgetragen worden sind. Eine Nachbehandlungsphase gab es nicht. In der Nachbehandlungsphase der aktuellen Untersuchungen wurde der antiinflammatorische Effekt von Dexpanthenol besonders ersichtlich.

Der repetitive Waschtest in der vorliegenden Form hat sich als geeignet erwiesen, die Hautschutzwirkung eines Präparates zu beurteilen und Aussagen über die Unterstützung von Repairmechanismen durch das Prüfpräparat zu machen. Dabei konnte ein Nutzeffekt von Dexpanthenol im Hinblick auf den hydratisierenden, barrierestabilisierenden und antiinflammatorischen Effekt gezeigt werden. 


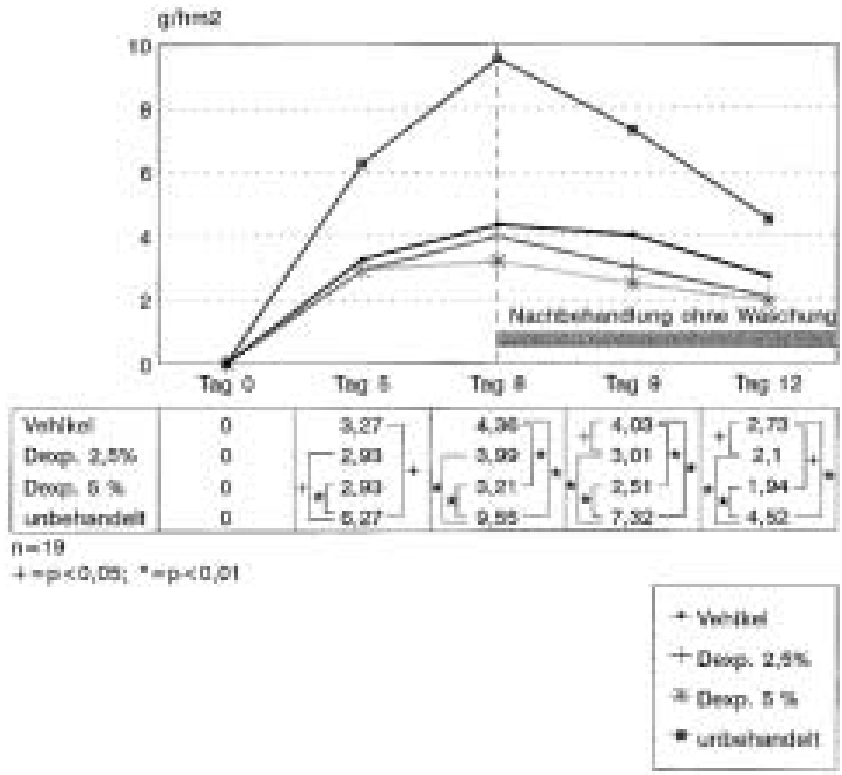

Abb. 4 Stabilisierender Einfluss von Dexpanthenol auf die epidermale Barrierefunktion (Tewameter).

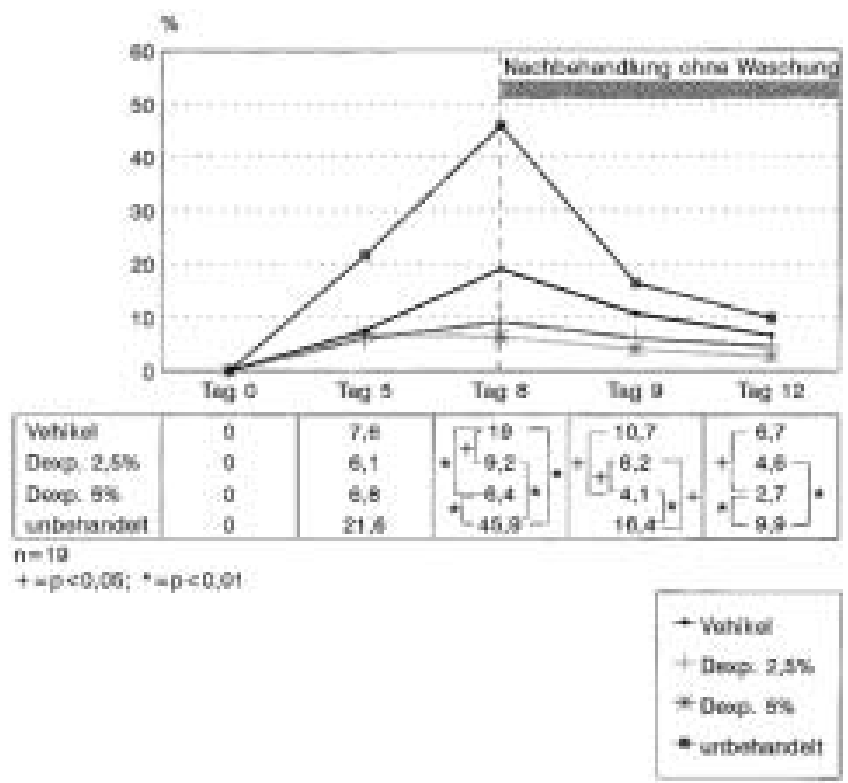

Abb. 5 Antiinflammatorische Wirkung von Dexpanthenol (LDF).

Die Ergebnisse sind auf empfindliche, atopische Haut übertragbar, da bei dieser Gruppe effektiver Hautschutz gegenüber hydrophilen Reizstoffen unabdingbar ist und bezüglich der Hornschichtfeuchtigkeit und der epidermalen Barrierefunktion ein vergleichbarer Zustand besteht, wie er durch die repetitiven Waschungen experimentell hervorgerufen wird [14].

Ein weiterer Aspekt unserer Untersuchungen ist die DosisWirkungs-Beziehung zwischen 2,5\% und 5\% Dexpanthenol. Im Hinblick auf den hydratisierenden Effekt und die stabilisierende Wirkung auf die epidermale Barrierefunktion sind 5\% Dexpanthenol gegenüber 2,5\% nur tendenziell, bezüglich der antientzündlichen Wirkung statistisch signifikant überlegen.

\section{Literatur}

${ }^{1}$ Berardesca E. EEMCO giudance for the assessment of stratum corneum hydration: electrical methods. Skin Res Technol 1997; 3: $126-132$

${ }^{2}$ Bettinger J, Gloor M, Gehring W, Wolf W. Influence of emulsions with and without urea on water-binding capacity of the stratum corneum. J Soc Cosm Chem 1995; 46: 247-254

${ }^{3}$ Bircher A, De Boer EM, Agner T, Wahlburg E, Serup J. Guidelines for measurement of cutaneous blood flow by laser Doppler flowmetry. Contact Dermatitis 1994; 30: $65-72$

${ }^{4}$ Fartasch M, Schnetz E, Diepgen T. Charakterization of detergentinduced barrier alterations - effect of barrier sreams on irritation. J Invest Dermatol 1998; 3: 121 - 127

${ }^{5}$ Frosch PJ, Kurte A. Efficacy of skin barrier creams (IV). The repetitive irritation test with a set of 4 standard irritants. Contact Dermatitis 1994; 31: $161-168$

${ }^{6}$ Gehring W, Gloor M. Der Effekt von Dexpanthenol bei experimentell geschädigter Haut. Z Hautkr 2001; 76: 1 - 7

${ }^{7}$ Gehring W, Gloor M. Effekt of topically applied Dexpanthenol on epidermal barrier function and stratum corneum hydration. Drug Res 2000; 50: 659-663

${ }^{8}$ Gehring W, Gloor M, Kleesz P. Predictive Washing Test for Evaluation of the Individual Eczema Risk. Contact Dermatitis 1998; 39: $8-13$

${ }^{9}$ Gehring W, Fischer MJ, Gottfreund J, Gloor M. Effect of various additives on the skin tolerability of a wash solution. Dermatosen 1996; 44: 160 - 163

${ }^{10}$ Gehring W, Kemter K, Nissen HP, Gottfreund J, Gloor M. Vergleichende Untersuchungen zum entfettenden Einfluss einer Waschlösung. Z Hautkr 1995; 70: 643-648

${ }^{11}$ Gehring W, Geier J, Gloor M. Untersuchungen über die austrocknende Wirkung verschiedener Tenside. Derm Mschr 1991; 177: $257-264$

${ }^{12}$ Gehse M, Scheer T, Gehring W, Gloor M. Das Friseurekzem - Ergebnisse gutachterlicher Bewertung von 1984-1987. Z Hautkr 1989; 64: $172-178$

${ }^{13}$ Grunewald AM, Gloor M, Gehring W. Efficiency of barrier creams. In: Elsner P, Maibach HI (Eds). Irritant Dermatitis - New clinical and experimental aspects. Karger Verlag, 1995: 23:187-197

${ }^{14}$ Loden M. Biophysical properties of dry atopic skin and normal skin with special reference to effects of skin care products. Acta Derm Venereol Suppl (Stockh) 1995; 192: 1-48

${ }^{15}$ Malten KE. Thoughts on irritant contact dermatitis. Contact Dermatitis 1981; 7: 238-247

${ }^{16}$ Nilsson GE, Otto U, Wahlberg JE. Assessment of skin irritancy in man by laser-doppler-flowmetry. Contact Dermatitis 1982; 8: $401-406$

17 Nilsson GE. Measurement of water exchange through skin. Med Biol Eng Comput 1977; 15: 209-221

18 Pinnagoda J, Tupker RA, Agner T, Serup J. Guidelines for transepidermal water loss (TEWL) measurement. Contact Dermatitis 1990; 22: $164-178$

${ }^{19}$ Schrader KH, Rohr M. Tenside - Ihre Beurteilung hinsichtlich Wirkung und Nebenwirkungen. Euro Cosmetcis 1994; 1: 18-22

${ }^{20}$ Wienert V, Hegner G, Sick H. Ein Verfahren zur Bestimmung des relativen Wassergehaltes des Stratum corneum der menschlichen Haut. Arch Dermatol 1981; 270: 67-75

\section{Prof. Dr. W. Gehring}

Hautklinik am Klinikum der Stadt Karlsruhe GmbH Moltkestraße 120

76133 Karlsruhe 